\title{
Spectral analysis of the nuclear stellar population and gas emission in six nearby galaxies ${ }^{\star}$
}

\author{
M. F. Saraiva, E. Bica, M. G. Pastoriza, and C. Bonatto
}

Departamento de Astronomia, Instituto de Física, Universidade Federal do Rio Grande do Sul, Av. Bento Gonçalves, 9500 - CP 15051, 91501-970, Porto Alegre, RS - Brazil

Received 22 February 2001 / Accepted 1 June 2001

\begin{abstract}
Optical spectra were used to study the stellar population and gas emission, when present, in the central parts of the elliptical galaxies NGC 6958, NGC 6851 and ESO 185-G54, and the spiral galaxies NGC 5757, NGC 7412 and IC 4214. Stellar population properties were derived by comparing the equivalent widths of the absorption lines with those of galaxy templates built from star clusters, as well as by applying a method of stellar population synthesis based on the same star clusters. For the galaxies with emission lines, the underlying stellar population was subtracted to obtain the pure emission spectrum, and the resulting emission line intensities were measured to determine the mechanism responsible for the gas excitation. From this analysis we concluded that the galaxies have solar metallicity except the elliptical ESO 185-G54 that reached metallicity slightly above solar. The three ellipticals and the Sa galaxy IC 4214 have their nuclei dominated by old stellar populations, although IC 4214 appears to have some contribution from the young generation stars. Recent star formation $(t<500 \mathrm{Myr})$ is prominent in the nucleus of the SBb galaxy NGC 5757, and is also present to a lesser degree in the Sbc NGC 7412. In these two galaxies, the recent star formation is responsible for the observed Hil type emission lines, while IC 4214 has LINER emission. Among the ellipticals, only in NGC 6958 did we detect ionized gas, whose spectra is typical of LINERs.
\end{abstract}

Key words. galaxies: elliptical and lenticular - galaxies: spiral - galaxies: ISM - galaxies: nuclei - galaxies: stellar content

\section{Introduction}

NGC 6851, NGC 6958 and ESO 185-G54 are elliptical galaxies listed in Phillips et al. (1988) as having emission lines in their nuclear spectra. In a previous surface photometry study (Saraiva et al. 1999) using BVRI and $\mathrm{H} \alpha$ filters, where we tried to map the ionized gas in a sample of elliptical galaxies, we could not detect any significant amount of gas in NGC 6851, although we detected some ionized gas in the nucleus of NGC 6958 and some evidence of it in ESO 185-G54. We also found that the nuclear colours of the three galaxies are typical of old, red stellar populations, with no evidence of recent star formation. The source of the ionizing photons in elliptical galaxies is not well known (Binette et al. 1994). Precise determinations of the stellar content and type of emission spectra are essential to clarify this problem. The other three galaxies in our sample are spirals with nuclear emission lines. NGC 5757 and NGC 7412 have their nuclear

Send offprint requests to: M. F. Saraiva,

e-mail: fatima@if.ufrgs.br

* Based on observations made at the Observatório do Pico dos Dias, CNPq/Laboratório Nacional de Astrofísica, Brazil. spectra classified as nuclear H II regions by Véron-Cetty \& Véron (1986), and IC 4214 was classified as a LINER by Maia et al. (1987). NGC 5757 is a strong barred galaxy, with a prominent nuclear ring, and a bright, point-like nucleus (Malkan et al. 1998). Its nuclear colours, compared with models of starbursts superimposed on old stellar populations (Bica et al. 1990) indicate that the flux is dominated by a stellar population younger than $0.5 \mathrm{Gyr}$ (Saraiva Schröder et al. 1994a). The nuclear colours in NGC 7412 are not significantly bluer than those of "normal" galaxies, but are bluer than the surrounding colours (Saraiva Schröder et al. 1994b). IC 4214 has a nuclear ring, very bright in the $\mathrm{H} \alpha+[\mathrm{NII}]$ lines, with colours suggesting that it is composed of HII regions mixed with dust (Saraiva 1997). Concerning these galaxies, more details on the properties of the nuclear stellar population are necessary for comparisons with the observed colours, as well as to make a suitable continuum subtraction in order to obtain the pure emission spectra. Therefore, there are three points we want to examine with respect to the 6 galaxies: to determine their nuclear stellar content, clarify the existence of the emission lines in the doubtful cases, and the type of emission spectra they have. 
Table 1. Basic parameters for the galaxies.

\begin{tabular}{|c|c|c|c|c|c|c|c|c|}
\hline $\begin{array}{l}\text { Galaxy } \\
(1)\end{array}$ & $\begin{array}{l}\text { Type } \\
(2)\end{array}$ & $\begin{array}{l}B_{\mathrm{T}} \\
(3)\end{array}$ & $\begin{array}{c}V_{\mathrm{rad}}\left(\mathrm{km} \mathrm{s}^{-1}\right) \\
(4)\end{array}$ & $\begin{array}{c}M_{B} \\
(5)\end{array}$ & $\begin{array}{l}\text { scale } \\
(6)\end{array}$ & $\begin{array}{c}A_{B}(\mathrm{BH}) \\
\quad(7)\end{array}$ & $\begin{array}{c}A_{B}(\mathrm{Sch}) \\
\quad(8)\end{array}$ & $\begin{array}{c}E(B-V)_{\mathrm{f}} \\
(9)\end{array}$ \\
\hline N 5757 & $\mathrm{SBb}$ & $12.73^{a}$ & $\begin{array}{c}2630 \pm 26 \\
(2624 \pm 192)\end{array}$ & -19.70 & 148 & 0.340 & 0.379 & 0.088 \\
\hline N 6851 & $\mathrm{E}:$ & $12.66^{b}$ & $\begin{array}{c}3039 \pm 15 \\
(3027 \pm 127)\end{array}$ & -20.10 & 174 & 0.160 & 0.200 & 0.046 \\
\hline N 6958 & $\mathrm{E}+$ & $12.28^{b}$ & $\begin{array}{c}2715 \pm 13 \\
(2728 \pm 18)\end{array}$ & -20.24 & 155 & 0.090 & 0.194 & 0.045 \\
\hline N 7412 & $\mathrm{SABc}$ & $11.20^{c}$ & $\begin{array}{c}1717 \pm 5 \\
(1795 \pm 171)\end{array}$ & -20.44 & 103 & 0.000 & 0.050 & 0.012 \\
\hline I 4214 & $\mathrm{SABa}$ & $12.27^{d}$ & $\begin{array}{c}2310 \pm 2 \\
(2354 \pm 203)\end{array}$ & -20.18 & 151 & 0.240 & 0.255 & 0.059 \\
\hline E 185-G54 & $\mathrm{E} 2$ & $12.10^{b}$ & $\begin{array}{c}4434 \pm 26 \\
(4298 \pm 89)\end{array}$ & -21.48 & 252 & 0.140 & 0.280 & 0.066 \\
\hline
\end{tabular}

Description of columns:

(1) Galaxy designation from RC3 (Third Reference Catalogue of Bright Galaxies, de Vaucouleurs et al. 1991).

(2) Morphological type (RC3).

(3) Total $B$ magnitude, in ${ }^{a}$ Saraiva Schröder et al. (1994a), ${ }^{b}$ Saraiva et al. (1999), ${ }^{c}$ Saraiva Schröder et al. (1994b), ${ }^{d}$ Saraiva (1997).

(4) Heliocentric radial velocity from NED (NASA/IPAC Extragalactic Database NASA/IPAC Extragalactic Database, which is operated by the Jet Propulsion Laboratory California Institute of Technology, under contract with the National Aeronautics and Space Administration). For comparison, we list in the second line the heliocentric radial velocity derived from our spectra, using the absorption lines centered at $4301 \AA, 4861 \AA, 5175 \AA$, and $5890 \AA$.

(5) Absolute magnitude, calculated from the heliocentric velocity corrected for a dipole Virgo-centric flow following Tammann \& Sandage (1985), and assuming $H=80 \mathrm{~km} \mathrm{~s}^{-1} \mathrm{Mpc}^{-1}$.

(6) Spatial scale, in parsec/".

(7) Burstein-Heiles' (1984) value of the Galactic absorption, taken from NED. $A_{B}(\mathrm{BH})$ values are based on H I fluxes and galaxy counts.

(8) Schlegel et al.'s (1998) value of Galactic absorption, taken from NED. $A_{B}(\mathrm{Sch})$ values are based on $100 \mu \mathrm{m}$ fluxes.

(9) Foreground colour excess $E(B-V)_{\mathrm{f}}=A_{B}(\mathrm{Sch}) / 4.25$.

Table 2. Log of the observations.

\begin{tabular}{lccc}
\hline \hline Galaxy & Slit $\left({ }^{\prime \prime}\right)$ & Exposure $(\mathrm{s})$ & Resolution $(\AA)$ \\
\hline N 5757 & $4.7,6.3$ & $5 \times 1200,7 \times 1200$ & $17.2,22.4$ \\
N 6851 & $4.7,6.3$ & $4 \times 1200,7 \times 1200$ & $17.2,22.4$ \\
N 6958 & 6.3 & $4 \times 1200$ & 22.4 \\
N 7412 & $4.7,6.3$ & $4 \times 1200,7 \times 1200$ & $17.2,22.4$ \\
I 4214 & $4.7,6.3$ & $3 \times 1200,3 \times 1200$ & $17.2,22.4$ \\
E 185-G54 & 6.3 & $4 \times 1200$ & 22.4 \\
\hline
\end{tabular}

In this work we analyze the optical spectra of these 6 galaxies, to complement the previous photometric study, and to better understand the star forming history and the gas ionizing source. We used the stellar population method based on star cluster integrated spectra described in Bica \& Alloin (1986), and Bica (1988 - hereafter B88), to analyze the properties of the nuclear stellar population (Sect. 3), as well as those of the nuclear gas, whenever present (Sect. 4). We discuss the individual galaxies in Sect. 5, and provide the concluding remarks in Sect. 6 .

\section{Observations and data reduction}

The sample galaxies and their basic parameters are given in Table 1. The observations were carried out in July 1994 at the Observatório do Pico dos Dias, Brazil, with the $1.60 \mathrm{~m}$ telescope and the Cassegrain spectrograph. The detector was the GEC \#48 CCD, with $1152 \times 770$ pixels of $22.5 \times 22.5 \mu \mathrm{m}^{2}$, and a grating of $192 \AA / \mathrm{mm}$. Two spectrograph slits were used, with widths of $4.7^{\prime \prime}$ and $6.3^{\prime \prime}$. The corresponding resolutions were $\simeq 17 \AA$ and $\simeq 22 \AA$ respectively, as measured from the comparison lamp lines. The slit was always oriented along the east-west direction, centered on the nucleus of the galaxy. Its total length was $230^{\prime \prime}$, and the approximate spectral coverage was 3800 $8200 \AA$. At least four exposures of $1200 \mathrm{~s}$ were taken for each galaxy. Six spectrophotometric standard stars were observed for flux calibration. A log of the observations is given in Table 2.

The data reduction, consisting of bias subtraction, flat-fielding, sky subtraction, wavelength and photometric calibration, and extinction correction, was carried out using standard IRAF $^{1}$ routines. The cosmic rays were eliminated in the process of combining the different exposures, after the extraction. The extraction window varied

\footnotetext{
${ }^{1}$ IRAF - Image Reduction and Analysis Facility - is distributed by the National Optical Astronomy Observatories, which is operated by the Association of Research in Astronomy, Inc., under cooperative agreement with the National Science Foundation, USA.
} 


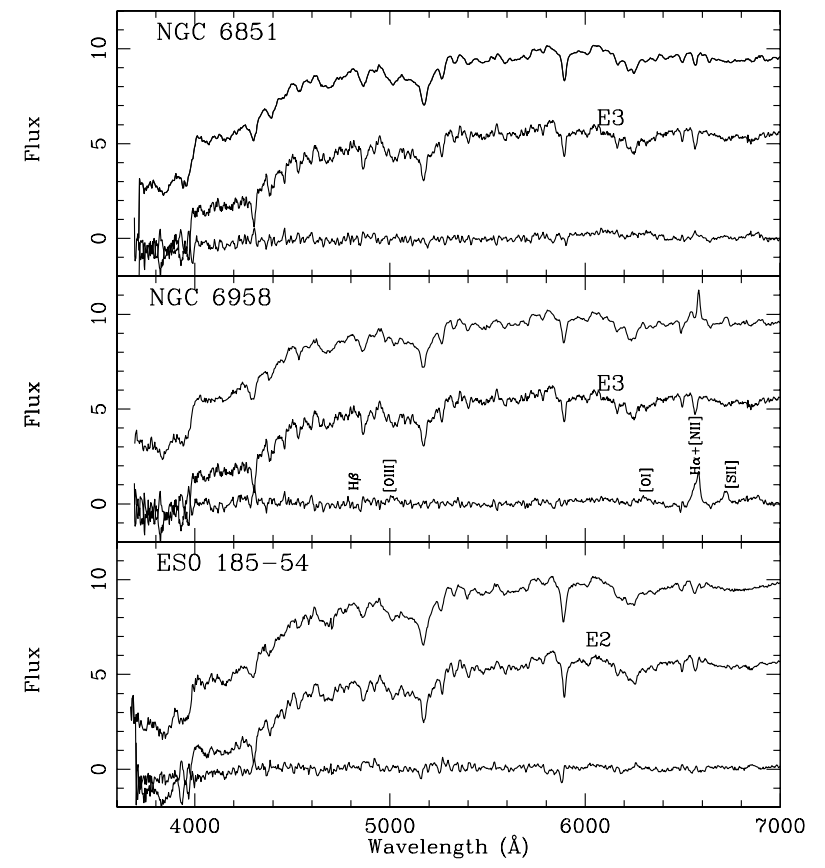

Fig. 1. Nuclear spectra of the elliptical galaxies. In each diagram, the top spectrum is the observed one, the middle spectrum (shifted by -4 ) is the matched template, and the residual spectrum is at bottom.

from $4^{\prime \prime}$ to $9^{\prime \prime}$ depending on the galaxy, in order to ensure extraction of a $2 \mathrm{kpc}$ region at the nucleus. Atmospheric absorption bands in the near infrared were removed by means of comparisons with hot standard stars (Bica \& Alloin 1987).

\section{Stellar population}

In order to study the stellar population content of nuclear regions of galaxies, we applied the stellar population analysis by B88, which is based on spectral group templates built from star clusters of different ages and metallicities, and nuclear galaxy spectral templates. Following this method, we measured the equivalent widths $(W)$ of the metal (K CaII, $C N$-band, $G$-band and $\mathrm{Mg}$ I) and Balmer $(\mathrm{H} \beta, \mathrm{H} \gamma$ and $\mathrm{H} \delta)$ lines. We did not use $\mathrm{H} \alpha$ because of possible emission line contamination. The measurements were made using a Gaussian fitting procedure of the IRAF reduction package, and are shown in Table 3. Mean errors in the equivalent widths for each spectrum were estimated measuring each line three times, allowing for differences in the continuum due to noise, and resulted to be $3 \%$ for strong lines $(W \geq 8 \AA)$ and $6 \%$ for the weak ones $(W<8 \AA)$. For galaxies with spectra in two different slits, we quoted in Table 3 the average values of the equivalent widths in both slits along with the standard deviation of the average. For NGC 5757 all Balmer lines contained important emission components, so we did not use them to study the stellar population.

The selection of the nuclear galaxy templates was based on the equivalent width of the absorption lines and continuum shape.

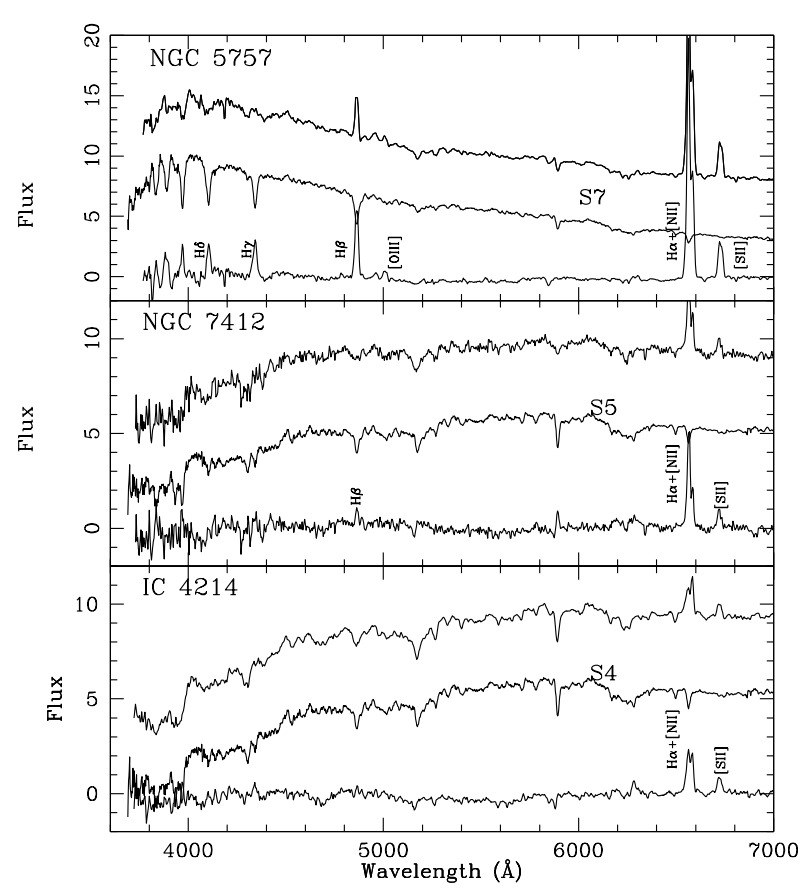

Fig. 2. Nuclear spectra of the spiral galaxies. In each diagram, top, middle and bottom spectra are as in Fig. 1.

To determine which template best fits each galaxy, we first compared the measured $W$ 's with those of the templates by searching for a minimum for the sum of the squared differences between the equivalent widths of galaxy and nuclear template. For the elliptical galaxies, we compared the present measurements with those of the E1 to E4 nuclear spectral template sequence, which is one of decreasing metallicity for essentially old stellar populations. We also tested the spectral groups E6, E7 and E8, which include increasing contributions from younger components. For the spiral galaxies, we used the spectral nuclear galaxy template sequences S1 to S7. The S1 to S3 templates form a sequence similar to that of E1 to E3 in the sense that they have decreasing metallicity at constant, mostly old, age. Groups S4 to S7 are subject to several age effects, forming a sequence in which the metallicity is almost constant, with increasing contribution from young stellar populations.

Next, we corrected the galaxy spectra for reddening using the foreground $E(B-V)_{\mathrm{f}}$ reddening values given in Table 1, and the extinction function of Cardelli et al. (1989). Subsequently we searched for the best match of the corrected spectrum to that of the selected template, or to a neighboring template in terms of properties. In all cases the template selected from the equivalent widths of the absorption lines also provided the best continuum distribution match, although for NGC 5757, NGC 6851 and ESO 185-G54 it was necessary to apply an additional reddening correction to improve this match, and for NGC 7412 it was necessary to subtract the applied reddening $E(B-V)_{\mathrm{f}}=0.012$ (Table 1 ). The extra reddening is possibly due to internal dust in the galaxies, but might also be due to zero point uncertainties in the 
Table 3. Equivalent widths of the main absorption lines.

\begin{tabular}{lccccccc}
\hline \hline \multirow{2}{*}{ Feature } & window & \multicolumn{3}{c}{$W(\AA)$} \\
\cline { 3 - 8 } & $\AA$ & $\mathrm{N} 5757$ & $\mathrm{~N} 6851$ & $\mathrm{~N} 6958$ & $\mathrm{~N} 7412$ & $\mathrm{I} 4214$ & E 185-G54 \\
\hline $\mathrm{KCa}$ II & $3908-3952$ & $2.6 \pm 0.28$ & $15 \pm 1$ & 15.6 & $7.5 \pm 1.3$ & $14.0 \pm 0.7$ & 16.5 \\
$\mathrm{H} \delta$ & $4082-4124$ & - & $3.9 \pm 0.4$ & 3.3 & $5.9 \pm 0.5$ & $5.9 \pm 0.9$ & 3.6 \\
$\mathrm{CN}$ & $4150-4214$ & $1.2 \pm 0.77$ & $7.6 \pm 0.4$ & 6.8 & $4.8 \pm 1.2$ & $8.0 \pm 0.5$ & 10.9 \\
$\mathrm{G}$ & $4284-4318$ & $1.7 \pm 0.12$ & $9.2 \pm 0.4$ & 6.0 & $5.3 \pm 0.6$ & $7.4 \pm 0.8$ & 8.4 \\
$\mathrm{H} \gamma$ & $4318-4364$ & - & $4.0 \pm 0.4$ & 2.5 & $3.1 \pm 1.2$ & $3.9 \pm 0.8$ & 3.3 \\
$\mathrm{H} \beta$ & $4846-4884$ & - & $3.0 \pm 0.1$ & 1.9 & $2.3 \pm 0.1$ & $3.1 \pm 0.3$ & 2.4 \\
$\mathrm{Mg}$ I & $5156-5196$ & $3.7 \pm 0.7$ & $8.8 \pm 0.7$ & 7.2 & $5.0 \pm 0.2$ & $7.1 \pm 0.04$ & 10.0 \\
$\mathrm{Na}$ II & $5880-5914$ & $2.2 \pm 0.7$ & $4.0 \pm 0.2$ & 2.3 & $3.3 \pm 1.5$ & $4.0 \pm 0.0$ & 5.9 \\
\hline
\end{tabular}

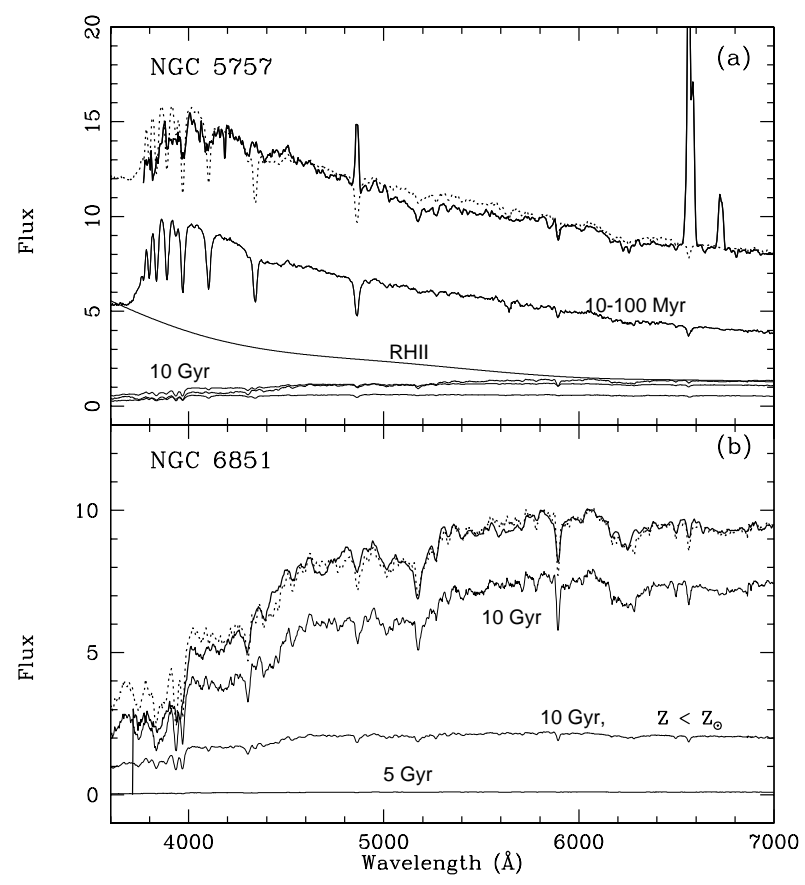

Fig. 3. a) Nuclear spectrum of NGC 5757 corrected for total reddening $E(B-V)=0.28$ (solid line) superposed to the synthesized one (dotted line). The remaining spectra (thin lines) are the synthesis components; we have labeled the most important ones according to their ages. b) Nuclear spectrum of NGC 6851 corrected for total reddening $E(B-V)=0.09$ (solid line) superposed to the synthesized one (dotted line). The thin lines are the components. In both diagrams, when not indicated, the stellar components have solar metallicity.

foreground reddening model. For instance, for NGC 7412, our adoptednecessary $E(B-V)=0$ is equal to the one calculated using Burstein-Heiles (1984) value for the Galactic absorption, instead of Schlegel et al.' (1998) (Table 1). On the other hand, for NGC 5757 nuclear dust probably accounts for all the extra reddening. In Table 4 we show the continuum ratios, the selected templates and the adopted reddening values.

Figures 1 and 2 show the absorption corrected spectra and the matched templates, as well as the residual spectra (galaxy - template).

The stellar population results were checked using an upgraded version (Schmitt et al. 1996; Raimann et al. 2000) of the star cluster synthesis method (B88), adapted
Table 4. Continuum ratios, selected templates and adopted colour excesses.

\begin{tabular}{lcccccc}
\hline \hline Galaxy & $\frac{3800}{5870}$ & $\frac{4020}{5870}$ & $\frac{4570}{5870}$ & $\frac{6630}{5870}$ & Template & $E(B-V)_{\mathrm{t}}$ \\
\hline N 5757 & 0.81 & 1.03 & 0.97 & 0.93 & S7 & 0.25 \\
N 6851 & 0.22 & 0.46 & 0.75 & 0.97 & E3 & 0.09 \\
N 6958 & 0.30 & 0.50 & 0.81 & 0.99 & E3 & 0.05 \\
N 7412 & 0.56 & 0.76 & 0.93 & 0.94 & S5 & 0.00 \\
I 4214 & 0.32 & 0.61 & 0.83 & 0.92 & S4 & 0.11 \\
E 185-G54 & 0.22 & 0.43 & 0.78 & 0.97 & E2 & 0.14 \\
\hline
\end{tabular}

to the optical region of the spectrum. The algorithm generates combinations of the spectral properties of the base elements, looking for the best match to the observed equivalent widths of specific absorption lines and relative fluxes of continuum points of a given galaxy spectrum.

The base elements for the synthesis are integrated spectra of star clusters of different ages and metallicities. We assume that the oldest population corresponds to globular clusters, to which we attribute an age of $10 \mathrm{Gyr}$. The method is particularly sensitive to mixture with younger components.

The employed lines are K CaII, $C N$-band, $G$-band and $\mathrm{MgI}$, and the continuum points are at $\lambda=3660$, 4020, 4510 and $6630 \AA$, normalized at $\lambda=5870 \AA$. Since the blue end of our spectra is $\lambda \simeq 3700 \AA$, we did not use the first point of the continuum that is usually employed in the synthesis, except in special cases. The synthesis solution must describe an evolutionary path in the plane age $\times\left[\frac{Z}{Z_{\odot}}\right]$, along which the metallicity varies from $\left[\frac{Z}{Z_{\odot}}\right]=-2$ to $\left[\frac{Z}{Z_{\odot}}\right]_{\max }$ at a fixed old age $(10 \mathrm{Gyr})$, and then the age varies down to $1 \mathrm{Myr}$ at constant metallicity. Three paths are allowed by the algorithm, reaching metallicities $\left[\frac{Z}{Z_{\odot}}\right]_{\max }=0.0,0.3$ and 0.6 respectively. A possible solution within a given path is found if the generated combination of the base elements (synthesized model) has equivalent widths of the absorption lines and relative fluxes of continuum points that reproduce those of the galaxy within allowed limits. The first approach is to look for a synthesis solution along the solar metallicity path, since we are dealing with the nuclear region (B88). If no solution is found along this path, then we try the remaining 
higher metallicity paths. We chose as best solution the synthesized spectrum that best matches the observed one.

The algorithm also takes into account the internal reddening $E(B-V)$ affecting the stellar population, varying it from 0.00 to 0.5 . For each reddening value, it searches for possible solutions among 84572315 combinations of the base elements (12 for most galaxies in the present study, Table 5). Assuming as the uniqueness parameter for the solutions the ratio (number of solutions)/(number of combinations), since typically we found 100 to 10000 solutions for each galaxy, the uniqueness parameter's value varies from $10^{-4}-10^{-2} \%$. In Table 5 we present the synthesis average solutions in the form of percentage flux contribution from each age and metallicity component to the light at $\lambda=5870 \AA$. For comparison, we quoted in parentheses the corresponding percentage contribution predicted by the matched template in Table 4.

The results from the synthesis are basically compatible with those from the templates, and in some cases, as for IC 4214 and NGC 5757, the agreement between the two procedures is fairly good. For NGC 7412 the synthesis attributed an exceedingly large contribution from the metal poor old population. For NGC 6851 and NGC 6958 the synthesized contribution from the old population with solar metallicity is larger than that predicted by the templates, and in the case of ESO 185-G54 it is smaller than that. Moreover, for this galaxy the small contribution from intermediate age population predicted by the matched template does not show up in the synthesized solution, which might be an indication of the uncertainty of the method in detecting stellar components with contributions smaller than $\simeq 10 \%$. The derived reddening from the two procedures is nearly the same.

Figure 3 shows the synthesized population for the galaxies NGC 5757 and NGC 6851, that have the bluest and reddest stellar populations respectively.

\section{Emission-line spectra}

The residual spectra (Figs. 1 and 2) obtained by the subtraction of the stellar population contribution defined by the matched template from the observed spectra, show that the three spiral galaxies and the elliptical NGC 6958 have nuclear emission lines.

For these galaxies, we compared the pure emission line spectra with the low emission galaxy nuclei spectra in Bonatto et al. (1989), and noted that NGC 5757 and NGC 7412 fits in Class A, corresponding to nuclear H II regions, NGC 6958 fits in class B, corresponding to LINERs, and IC 4214 between B and C; class C corresponds to objects with lower excitation and lower emission level than classical LINERs. In order to have a more quantitative classification of the galaxies, we measured the emission line intensities and used the diagnostic diagrams $[\mathrm{O}$ III $] \lambda 5007 / \mathrm{H} \beta$ versus $[\mathrm{N} \mathrm{II}] \lambda 6583 / \mathrm{H} \alpha,[\mathrm{O}$ III $\lambda$ 5007/H $\beta$ versus $[\mathrm{S} \mathrm{II}] \lambda 6716+\lambda 6731 / \mathrm{H} \alpha$, and $[\mathrm{O}$ III $\lambda 5007 / \mathrm{H} \beta$ versus [O I] $\lambda 6300 / \mathrm{H} \alpha$, from Veilleux \& Osterbrock (1987).
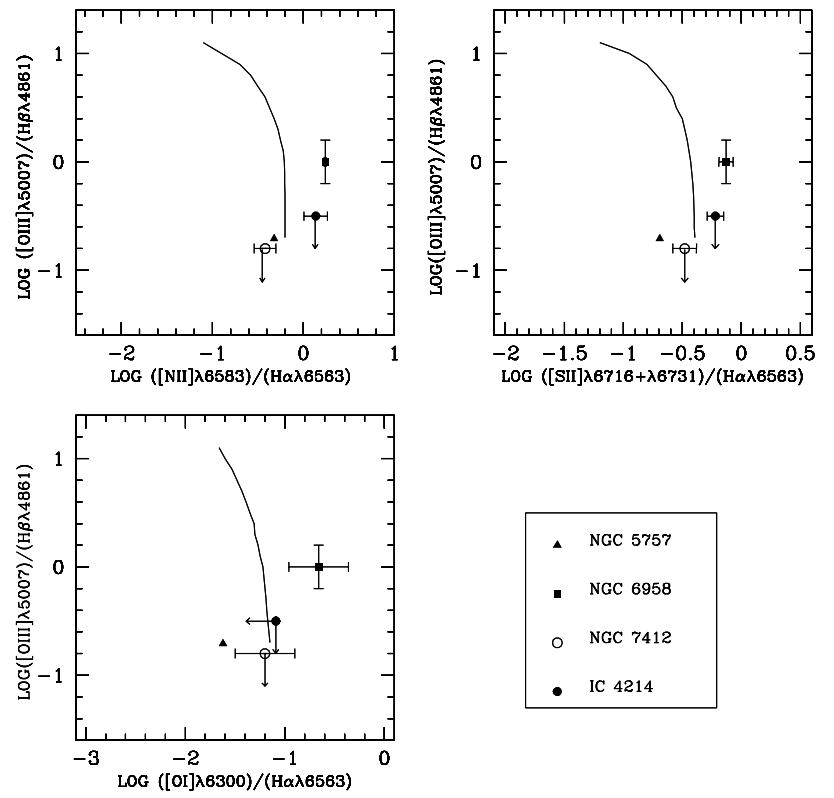

Fig. 4. Intensity line-ratios plotted in the diagnostic diagrams from Veilleux \& Osterbrock (1980). The continuous line is the curve separating AGNs from $\mathrm{H}$ II region like objects. The arrows indicate upper limits; for NGC 5757 the errors are smaller than the symbol.

The measurements of the fluxes and equivalent widths of the lines were carried out in the same manner for the absorption lines, using Gaussian fitting. The procedure allowed us to de-blend the line $\mathrm{H} \alpha+[\mathrm{N} \mathrm{II}]$.

The fluxes were reddening corrected using the $E(B-V)$ value obtained assuming the case $\mathrm{B}$ of the recombination theory (Osterbrock 1989), and Seaton reddening law (Seaton 1979).

The measurements are shown in Table 6, where we also list the calculated $\mathrm{H} \alpha$ luminosities. Colons indicate flux ratios with uncertainties around $50 \%$. For very weak lines we quoted only upper limits. The diagnostic diagrams are shown in Fig. 4.

\section{Results}

Below we discuss the results for each galaxy separately:

NGC 5757: This is the galaxy with the bluest nucleus $(\mathrm{C} 4020 / \mathrm{C} 5870=1.45$ after the reddening correction $)$ and the highest $\mathrm{H} \alpha$ luminosity in our sample. The results of the stellar population analyses indicate that the stellar population is composed predominantly (around $70 \%$ of the flux at $5870 \AA$ ) by young stars (age $\leq 500 \mathrm{Myr}$ ) of solar metallicity $\left(\left[Z / Z_{\odot}\right]=0\right)$. The continuum fitting required a reddening correction $E(B-V)=0.25-0.28$, twice or more Schlegel et al.'s value. A similar value $\left[E(B-V)_{\text {gas }}=0.27\right]$ was derived from the emission line ratio $\mathrm{H} \alpha / \mathrm{H} \beta$. The diagnostic diagrams show that the pure emission spectrum is typical of nuclear $\mathrm{H}$ II regions.

NGC 6851: The template that best matches the absorption spectrum of this galaxy is E3, suggesting that the bulk $(\simeq 70 \%)$ of the stellar population has attained 
Table 5. Population components.

\begin{tabular}{|c|c|c|c|c|c|c|c|c|c|c|}
\hline \multirow[t]{2}{*}{ Galaxy } & \multirow[t]{2}{*}[Z/Z_{\odot}]{} & \multicolumn{8}{|c|}{ Age } & \multirow{2}{*}{$\begin{array}{c}E(B-V) \\
\pm \sigma\end{array}$} \\
\hline & & HII & $10^{7}$ & $5 \times 10^{7}$ & $10^{8}$ & $5 \times 10^{8}$ & $10^{9}$ & $5 \times 10^{9}$ & $10^{10}$ & \\
\hline N 5757 & $\begin{array}{c}0.0 \\
-0.5 \\
-1.0 \\
-1.5 \\
-2.0 \\
\end{array}$ & $16(12)$ & $10(10)$ & $10(11)$ & $32(32)$ & $2(7)$ & $2(2)$ & $2(3)$ & $\begin{aligned} & 14(17) \\
& 5(2) \\
& 3(2) \\
& 2(1) \\
& 2(1) \\
&\end{aligned}$ & $\begin{array}{c}0.28 \\
0.282\end{array}$ \\
\hline N 6851 & $\begin{array}{c}0.0 \\
-0.5 \\
-1.0 \\
-1.5 \\
-2.0\end{array}$ & $0(0)$ & $0(0)$ & $0(0)$ & $0(0)$ & $0(0)$ & $0(0)$ & $1(5)$ & $\begin{array}{c}78(64) \\
12(14) \\
5(11) \\
3(4) \\
1(2)\end{array}$ & $\begin{array}{c}0.08 \\
0.077\end{array}$ \\
\hline N 6958 & $\begin{array}{c}0.0 \\
-0.5 \\
-1.0 \\
-1.5 \\
-2.0 \\
\end{array}$ & $0(0)$ & $1(0)$ & $0(0)$ & $1(0)$ & $2(0)$ & $4(0)$ & $7(5)$ & $\begin{array}{c}77(64) \\
5(14) \\
2(11) \\
1(4) \\
0(2) \\
\end{array}$ & $\begin{array}{c}0.07 \\
0.077\end{array}$ \\
\hline N 7412 & $\begin{array}{c}0.0 \\
-0.5 \\
-1.0 \\
-1.5 \\
-2.0 \\
\end{array}$ & $9(6)$ & $1(2)$ & $0(2)$ & $0(2)$ & $0(1)$ & $0(2)$ & $1(4)$ & $\begin{array}{c}43(51) \\
11(12) \\
7(7) \\
11(6) \\
18(5) \\
\end{array}$ & $\begin{array}{c}0.03 \\
0.035\end{array}$ \\
\hline I 4214 & $\begin{array}{c}0.0 \\
-0.5 \\
-1.0 \\
-1.5 \\
-2.0 \\
\end{array}$ & $1(1)$ & $2(1)$ & $1(1)$ & $2(2)$ & $2(1)$ & $3(3)$ & $5(9)$ & $\begin{array}{c}63(65) \\
11(7) \\
5(5) \\
3(3) \\
2(2) \\
\end{array}$ & $\begin{array}{c}0.15 \\
0.151\end{array}$ \\
\hline E 185-G54 & $\begin{array}{c}+0.3 \\
0.0 \\
-0.5 \\
-1.0 \\
-1.5 \\
-2.0\end{array}$ & $0(0)$ & $0(0)$ & $0(0)$ & $0(0)$ & $0(0)$ & $0(0)$ & $1(11)$ & $\begin{array}{c}53(74) \\
28(6) \\
9(4) \\
4(3) \\
3(1) \\
2(1)\end{array}$ & $\begin{array}{c}0.11 \\
0.114\end{array}$ \\
\hline
\end{tabular}

Table 6. Emission line fluxes.

\begin{tabular}{|c|c|c|c|c|c|c|c|c|c|}
\hline \multirow[t]{2}{*}{ Galaxy } & \multicolumn{6}{|c|}{ Flux ratios $\left(\frac{F_{\lambda}}{F_{\mathrm{H} \alpha}}\right)$} & \multirow{2}{*}{$\begin{array}{c}F_{\mathrm{H} \alpha} \\
\text { (a) }\end{array}$} & \multirow{2}{*}{$\begin{array}{c}L_{\mathrm{H} \alpha} \\
\text { (b) }\end{array}$} & \multirow{2}{*}{$\begin{array}{c}E(B-V)_{\text {gas }} \\
\text { (c) }\end{array}$} \\
\hline & $\begin{array}{c}\mathrm{H} \beta \\
\lambda 4861\end{array}$ & $\begin{array}{l}\text { O III] } \\
\lambda 5007\end{array}$ & $\begin{array}{c}\mathrm{O} \text { I }] \\
\lambda 6300\end{array}$ & $\begin{array}{c}{[\mathrm{N} \text { II }]} \\
\lambda 6548\end{array}$ & $\begin{array}{c}{[\mathrm{N} \text { II }]} \\
\lambda 6584\end{array}$ & $\begin{array}{c}\mathrm{S} \text { II }] \\
\lambda 6717+6731\end{array}$ & & & \\
\hline N 5757 & 0.27 & 0.053 & 0.023 & 0.15 & 0.467 & 0.20 & 35 & 35.5 & 0.27 \\
\hline N 6958 & 0.18 & $0.31:$ & 0.18 & 0.42 & 1.789 & 0.74 & 4 & 5.1 & 0.59 \\
\hline N 7412 & 0.17 & $<0.03$ & 0.068 & $0.18:$ & 0.287 & 0.375 & 2.5 & 1.3 & 0.63 \\
\hline I 4214 & 0.144 & $<0.06$ & $<0.07$ & 0.21 & 1.3 & 0.55 & 8 & 8.6 & 0.72 \\
\hline
\end{tabular}

(a) $\mathrm{H} \alpha$ fluxes in units of $10^{-14} \mathrm{ergs} \mathrm{s}^{-1} \mathrm{~cm}^{-2}$.

(b) $\mathrm{H} \alpha$ luminosities in units of $10^{39} \mathrm{ergs} \mathrm{s}^{-1}$.

(c) Color excesses calculated from the relation $E(B-V)=-2.15 \log \left(\frac{2.8}{F_{\mathrm{H} \alpha} / F_{\mathrm{H} \beta}}\right)$.

the solar metallicity, and about $95 \%$ of the stars are older than $10 \mathrm{Gyr}$, with a very small contribution $(\simeq 5 \%)$ of an intermediate age population $(t \simeq 5 \mathrm{Gyr})$. The synthesis gives a smaller contribution from this intermediate age population, but comparable within the uncertainties.

In spite of the measurements by Phillips et al. (1988) and Macchetto et al. (1996), we could not detect any emission line in this galaxy's nucleus.
NGC 6958: The equivalent widths of the absorption lines and continuum shape indicate that the best match for this galaxy is also the template E3, and the population derived by the synthesis algorithm agrees very well with this template's components. Therefore this galaxy has basically the same type of stellar population as NGC 6851, where intermediate age contributions are very small or negligible. NGC 6958 is the only elliptical galaxy in our 
sample whose residual spectrum [(observed spectrum) (matched template) presents emission lines. The measured flux of the line $[\mathrm{N}$ II $] \lambda 6584 \AA$ is in agreement with that measured by Phillips et al. (1988). The location of the flux ratios of the emission lines in the diagnostic diagrams indicates a LINER type emission nuclear spectrum. The gas reddening derived from the $\mathrm{H} \alpha / \mathrm{H} \beta$ ratio is $E(B-V)_{\text {gas }}=0.6$, much higher than the value obtained for the stellar population $[E(B-V)=0.05-0.07]$. This indicates that, in this particular early type galaxy, the dust is more heavily concentrated in the emitting region, not affecting significantly the light from the stars, as shown by extinction maps in large samples of early type galaxies (Ferrari et al. 1999; Goudfrooij et al. 1994). Alternatively, the $\mathrm{H} \alpha / \mathrm{H} \beta$ ratio could not be due to a pure recombination spectra. High spatial resolution spectroscopy is necessary in order to determine unambiguously the cause of this effect.

NGC 7412: The template that best matches the equivalent widths and continuum of this Sbc galaxy is S5, whose stellar content is dominated $(70 \%)$ by stars that reached solar metallicity, among which $13 \%$ have ages between $10^{7}$ and $5 \times 10^{9}$ years, and $6 \%$ are younger than $10^{7}$ years. The synthesis agrees with this template with respect to the solar metallicity old component, but owing to some spectral degeneracy, the algorithm tends to prefer old metal poor components rather than young ones (10 to $500 \mathrm{Myr}$ ), and to a lesser extent intermediate age components. A small contribution from a $10^{6}$ years component is also found. The emission spectrum is typical of nuclear $\mathrm{H}$ II regions. The $\mathrm{H} \alpha / \mathrm{H} \beta$ ratio indicates that the gas is reddened by an amount $E(B-V)_{\text {gas }}=0.63$, compared with the stellar reddening $E(B-V)=0.0-0.03$. This again suggests localized dust in star forming regions.

IC 4214 This is the galaxy for which the synthesis results best agree with the matched template, S4. The bulk $(80 \%)$ of the stellar population has reached solar metallicity, and more than $80 \%$ is older than 1 Gyr. Among the three spirals of the sample, this galaxy is the only one with minimal star formation in the nucleus. This galaxy has a blue nuclear ring (as mentioned in Sect. 1), and part of this nuclear ring is included in our slit. Therefore, the contribution from a possible young stellar component $(t \leq 500 \mathrm{Myr})$ present in the nuclear ring is very small compared with the strong concentration of old stars at the center. The stellar internal reddening is around 0.05-0.09, as compared with the gas internal reddening $E(B-V)_{\text {gas }}=0.7$, so the gas appears to be localized. The diagnostic diagram confirms that the emission lines are typical of LINERs.

ESO 185-G54: The nuclear spectrum of this galaxy indicates it is dominated by a very old ( $t>10 \mathrm{Gyr}$ ) population. It differs from the other two ellipticals in the sense that it is the only one reaching metallicity slightly above solar $\left(\left[Z / Z_{\odot}\right]=0.3\right)$. Although the template E2, that best fits this galaxy, has a $10 \%$ component of intermediate age population, this component does not appear in the synthesis, unless we select solutions with very high colour excess. No emission line was detected.

\section{Concluding remarks}

From the present spectroscopy study we conclude that:

The three elliptical galaxies have nuclear stellar content substantially older than the spiral ones, but there is no clear division between the two groups in terms of metallicity. All the studied galaxies reach solar metallicity, except ESO 185-G54, that reaches metallicity twice solar. Recent star formation is present prominently in the nucleus of the SBb galaxy NGC 5757 and to a lesser degree in the nucleus of the Sbc NGC 7412. The dominant nuclear stellar population in each galaxy is consistent with their broad band colours.

Among the three elliptical galaxies cataloged as having nuclear emission lines, for only one of them, NGC 6958, did we detect this emission. This emphasizes the importance of spectral observations and a correct determination of the stellar population to obtain an accurate continuum subtraction. Ionized gas is also present in the three spiral galaxies.

For three galaxies out of the four that present emission lines (NGC 6958, NGC 7412 and IC 4214) the gas reddening determined by the $\mathrm{H} \alpha / \mathrm{H} \beta$ ratio is much larger than that derived from the continuum spectra, probably indicating that the dust is concentrated in the emitting region.

The position of the galaxies in diagnostic diagrams based on emission line flux ratios confirms the previous classification of NGC 5757 and NGC 7412 as H II region nuclei, and IC 4214 as LINER. The elliptical NGC 6958 also fits in the LINER category. This is in agreement with the more frequent nuclear emission types in spirals and early type galaxies, respectively (Bonatto et al. 1989).

Acknowledgements. We are very grateful to D. Raimann for giving us the upgraded version of the stellar population synthesis computer program, and kindly assisting us in its use. Claudia Winge, Alberto Ardila and Carlos Dutra were also very helpful with the initial reductions; M. F. S. thanks the student Ricardo Cunha da Silva for working in this project. This work was in part supported by PRONEX/FINEP 76.97.10003.00 and $\mathrm{CNPq}$.

\section{References}

Bica, E., \& Alloin, D. 1986, A\&A, 66, 171

Bica, E., \& Alloin, D. 1987, A\&A, 186, 49

Bica, E. 1988, A\&A, 195, 76 (B88)

Bica, E., Alloin, D., \& Schmidt, A. 1990, MNRAS, 242, 241

Binette, L., Magris, C. G., Stasińska, G., \& Bruzual, A. G. 1994, A\&A, 292, 13

Bonatto, C., Bica, E., \& Alloin, D. 1989, A\&A, 226, 23

Burstein, D., \& Heiles, C. 1984, ApJ, 225, 40

Cardelli, J. A., Clayton, G. C., \& Mathis, J. S. 1989, ApJ, 345, 245

de Vaucouleurs, G., de Vaucouleurs, A., Corwin, H. G., et al. 1991, The Third Reference Catalogue of Bright Galaxies (Springer Verlag, New York) (RC3) 
Goudfrooij, P., Hansen, L., Jørgensen, H. E., \& NørgaardNielsen, H. U. 1994, A\&AS, 105, 41

Saraiva Schröder, M. F., Pastoriza, M. G., \& Kepler, S. O. 1994a, A\&AS, 104, 487

Macchetto, F., Pastoriza, M., Caon, N., et al. 1996, A\&AS, Saraiva Schröder, M. F., Pastoriza, M. G., Kepler, S. O., \& 120,463 Puerari, I. 1994b, A\&AS, 108, 41

Maia, M. A. G., da Costa, L. N., Willmer, C., Pellegrini, P. S., Saraiva, M. F. 1997, AJ, 113, 1607 \& Rité, C. 1987, AJ, 93, 546

Malkan, M. A., Gorjian, V., \& Tam, R. 1998, ApJS, 117, 25

Osterbrock, D. E. 1989, Astrophysics of Gaseous Nebula and Active Galactic Nuclei (Univ. Science Books, Mill Valley)

Phillips, M. M., Jenkins, C. R., Dopita, M. A., Sadler, E. M., \& Binette, L. 1988, AJ, 91, 1062

Raimann, D., Bica, E., Storchi-Bergmann, T., Melnick, J., \& Schmitt, H. 2000, MNRAS, 314, 295

Sandage, A., \& Tammann, G. 1981, A Revised Shapley-Ames Catalogue of Bright Galaxies (Carnegie Inst. of Washington Pub.), No. 635 (RSA)

Saraiva, M. F., Ferrari, F., \& Pastoriza, M. G. 1999, A\&A, 350,399

Schlegel, D. S., Finkbeiner, D. P., \& Davis, M. 1998, ApJ, 500, 525

Schmitt, H. R., Bica, E., \& Pastoriza, M. G. 1996, MNRAS, 278,965

Seaton, M. J. 1979, MNRAS, 187, 73

Tammann, G. A., \& Sandage, A. 1985, ApJ, 294, 81

Veilleux, S., \& Osterbrock, D. 1987, ApJS, 63, 295

Véron Cetty, M. P., \& Véron, P. 1986, A\&AS, 66, 335 